\title{
Low-mass stellar models with new opacity tables and varying $\alpha$-element enhancement factors
}

\author{
A. Weiss ${ }^{1}$, J. Ferguson ${ }^{2}$ and M. Salaris ${ }^{3}$ \\ ${ }^{1}$ Max-Planck-Institut für Astrophysik, Karl-Schwarzschild-Str. 1, 85748 Garching, Germany \\ email: aweiss@mpa-garching.mpg.de \\ ${ }^{2}$ Astrophysics Research Institute, Liverpool John Moores University, \\ Twelve Quays House, Egerton Wharf, Birkenhead, CH41 1LD, UK \\ ${ }^{3}$ Physics Department, Wichita State University, Wichita, KS 67260-0032, USA
}

\begin{abstract}
We have computed new models for stars of low and intermediate mass, with varying degrees of $\alpha$-element enhancement factors, using new low-temperature molecular opacities. We present some of the effects found. $\dagger$
\end{abstract}

Keywords. stars: general, evolution, low-mass

\section{Motivation}

The increasing demands of stellar population synthesis for more accurate stellar models has led to the necessity for a fully self-consistent treatment of chemical compositions in the models. While we are far from considering any mixture correctly, the current development is for consistent models and spectra for $\alpha$-enhanced compositions. One such example is presented by P. Coelho in this meeting. For this project we calculated new stellar models for both solar-scaled and $\alpha$-enhanced compositions of varying total metallicity. For the $\alpha$-elements a constant enhancement of 0.4 dex was chosen. We produced solar and $\alpha$-enhanced models for three $[\mathrm{Fe} / \mathrm{H}]$-values. For $[\mathrm{Fe} / \mathrm{H}]=-0.5$ the two mixtures are $X=0.743, Z=0.032$ (solar) and $X=0.739, Z=0.011$ ( $\alpha$-enhanced). At $[\mathrm{Fe} / \mathrm{H}]=0.0$ we have $(X, Z)=(0.718,0.017)$ resp. $(0.679,0.032)$, and for $[\mathrm{Fe} / \mathrm{H}]=0.2$ finally $(X, Z)=0.708,0.026$ (solar) and $(0.642,0.048)$ ( $\alpha$-enhanced).

So far, models between $M=0.6 M_{\odot}$ and $10 M_{\odot}$ have been calculated. We followed the evolution from the MS to the RGB tip, resp. the early AGB, resp. the end of core He burning.

\section{Opacity effects}

Opacity tables are not always available for the exact model composition. We had available low-temperature tables with varying internal $\alpha$-element enhancements as in Salaris \& Weiss (1998), calculated with the code by Alexander \& Ferguson (1994), but also new ones for the constant enhancement factors given above. The latter were specifically produced with the code of Ferguson et al. (2005). Consistent high-T OPAL tables (Iglesias \& Rogers 1996) were combined with these. This allowed us to investigate the influence of varying degrees of individual $\alpha$-element enhancements on the models. Fig. 1 (left panel) shows the comparison of evolutionary tracks for stars with mass between 0.6 and $1.3 M_{\odot}$ with either variable (case A; solid) or constant (case B; dotted lines) $\alpha$-enhancements.

$\dagger$ The extended version of this paper is available as astro-ph/0605666 

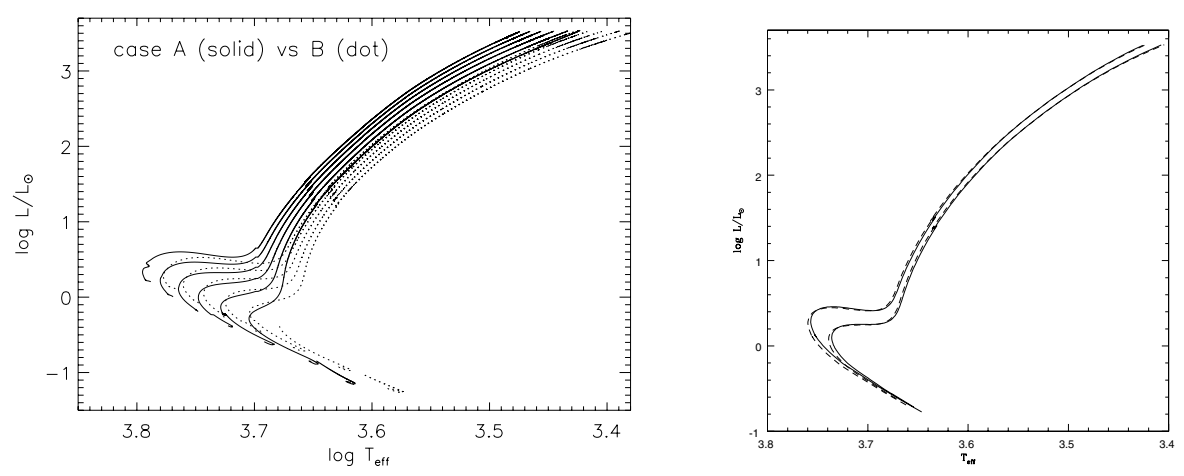

Figure 1. Left: Stellar models for $X=0.679, Z=0.032$ using opacity tables with either variable (solid) or constant (dotted lines) $\alpha$-element enhancement factors. Right: Isochrones for 8 and 14 Gyr for models with $Z=0.032$, fully self-consistently calculated, including all opacity tables for a variable (dashed) or constant (solid; slightly lower $T_{\text {eff }}$ at the turn-off) $\alpha$-enhancement.

There were surprisingly large differences between the two sets of models: (i) the "variable $\alpha$ " tables resulted in much warmer $T_{\text {eff }}$ (up to $+250 \mathrm{~K}$ ), and (ii) the lifetimes were up to $15 \%$ shorter than in the "constant $\alpha$ " case. It turned out that theses two effects can be ascribed separately to (i) the low-T opacity tables of 1994, producing the higher temperatures, and (ii) the chemical composition of the high- $\mathrm{T}$ tables, which results in consistently higher opacities for temperatures between $10^{4}$ and $10^{7} \mathrm{~K}$.

The first effect is due an error in the production of these specific low-T tables in 1994, the second one is real as was confirmed by test calculations using Opacity Project data. With the new code by Ferguson et al. (2005) new "variable $\alpha$ " tables have been produced, which agree well with those for constant $\alpha$-enhancement; $T_{\text {eff }}$ differences are reduced to the $10 \mathrm{~K}$ level or below. The evolutionary age differences at given mass remain.

\section{Consequences}

The error in the old low- $T$ opacity tables becomes significant for $Z \gtrsim 0.5 Z_{\odot}$; models which used these specific tables should be recalculated with corrected tables (e.g. Salasnich et al. 2000). For lower $Z$ the effect vanishes, and the tracks are no longer affected; work on globular clusters, such as Salaris \& Weiss (1998), remains valid. Only the low-T tables of our group were affected; it is not an error in the Alexander \& Ferguson (1994) code. The influence of individual element abundances on stellar lifetimes is real, but isochrones are almost identical in their HRD-location (Fig. 1, right panel), although TO-masses differ by about $0.05 M_{\odot}$. For accurate stellar parameters, including mass and age, all element abundances may prove significant, in particular that of oxygen.

\section{References}

Alexander, D. R. \& Ferguson, J. W. 1994, ApJ, 437, 879

Ferguson, J. W., Alexander, D. R., Allard, F., et al. 2005, ApJ, 623, 585

Grevesse, N. \& Sauval, A. J. 1998, Space Sci. Rev., 85, 161

Iglesias, C. A. \& Rogers, F. J. 1996, ApJ, 464, 943

Salaris, M. \& Weiss, A. 1998, A\&A, 335, 943

Salasnich, B., Girardi, L., Weiss, A., \& Chiosi, C. 2000, A\&A, 361, 1023 